\title{
Reversible icosahedral-rhombohedral transition related to phason effects in an AlFeCu alloy
}

\author{
Marc Audier \\ Laboratoire de Thermodynamique et Physico-Chimie Métallurgiques (UA CNRS $n^{\circ} 29$ ) ENSEEG, \\ BP 75, 38402 Saint Martin d'Hères, France
}

(Received October 01, 1990; accepted February 01, 1991)

\begin{abstract}
Résumé. - Les différents états structuraux, rhomboédrique, icosaédrique et modulé, que peuvent présenter des particules dodécaédriques d'alliage $\mathrm{Al}_{63,5} \mathrm{Fe}_{12,5} \mathrm{Cu}_{24}$ ont été caractérisés par microscopie électronique à haute résolution. Le quasi-réseau d'Ammann, susceptible de caractériser la phase icosaèdrique a un paramètre de $4,46 \tau^{4} \AA$. Des modèles bidimensionnels, déduits des observations, permettent d'interpréter la transition à partir de phasons.
\end{abstract}

Abstract. - Different states, rhombohedral, icosahedral and modulated, of dodecahedral particles of the $\mathrm{Al}_{63.5} \mathrm{Fe}_{12.5} \mathrm{Cu}_{24}$ alloy have been characterized by High Resolution Electron Microscopy. Whether of perfect structure, the icosahedral phase can be related to 3D-Ammann quasilattice of parameter $4.46 \tau^{4} \AA$. Two-dimensional models, directly related to HREM results, are presented in order to interpret the phase transition from phasons.

\section{Introduction.}

A large variety of quasicrystalline intermetallic compounds have been identified since their discovery was reported in 1984 by Shechtman, Blech, Gratias and Cahn [1]. Among these ones, some, like Al-Cu-Fe, $\mathrm{Al}-\mathrm{Cu}-\mathrm{Mn}$ and $\mathrm{Al}-\mathrm{Cu}-\mathrm{Ru}$ [2] have been found to be of perfect icosahedral structure, or nearly, exhibiting the particularity to be typical of an ordered superstructure of the usual primitive quasilattice [ $3-5]$. Considered, at present, as representative of ideal quasicrystals, such materials are the focus of a number of experimental and theoretical studies and it can be expected that fundamental answers will be soon obtained about the exact nature of perfect icosahedral phases, i.e. characteristic of either a deterministic or random tiling and which differences of diffracting properties and thermodynamical stability have been theoretically established [6]. For instance, the results of Calvayrac et al. [7], indicating that a thermodynamical stability of the $\mathrm{Al}-\mathrm{Fe}-\mathrm{Cu}$ icosahedral phase, between 0 Kelvin and its melting point temperature, may be plausible, it can be assumed that such an icosahedral phase could be rather characteristic of a deterministic quasilattice. 
Apparently at variance with this idea, the occurence of a reversible icosahedral-rhombohedral transition in an $\mathrm{Al}_{63.5} \mathrm{Fe}_{12.5} \mathrm{Cu}_{24}$ alloy has been recently identified by transmission electron microscopy [8]. This phase transition has also been observed by X-ray [9] and neutron diffraction [10], thus supporting entropic stabilization models [11]. As results, we have found that at low temperature, the stable state is rhombohedral with a microcrystalline structure constituted of small domains exhibiting special crystallographical orientational relationships imparting an overall pseudo-icosahedral symmetry. At high temperature, this micro-domain structure transforms homogeneously into an icosahedral phase which is stable up to melting [12]. The structural transformation is reversible and occurs via different transient states, the most spectacular one being defined as a modulated icosahedral phase. An interpretation of the phenomenon leading to a modulated quasicrystalline structure has been presented on the basis of phason theory [6] applied to the simple case of a one-dimensional aperiodic chain of Fibonnaci [13].

Here, we report further HREM characterizations about the different states of this transformation, namely those of the microcrystalline, icosahedral and modulated structures. On the basis of previous results and from a careful examination of HREM images, we deduce the edge length of the Ammann rhombohedra which could be considered as constituting the quasilattice of a perfect icosahedral state, as well as some characteristics of their decoration. From two-dimensional models representing the projection of the quasicrystalline and crystalline structures, such they are observed by HREM, we check that phasons can be effectively involved in this phase transition.

Samples of $\mathrm{Al}_{63.5} \mathrm{Fe}_{12.5} \mathrm{Cu}_{24}$ were prepared by melting the pure elements at $1200^{\circ} \mathrm{C}$ under argon atmosphere and then solidified under vacuum at cooling rate slower than $1^{\circ} \mathrm{C} \mathrm{mn}^{-1}$, down to room temperature. When broken open, the ingots exhibited particles of dodecahedral shape inside their shrink cavities. Extracted from ingots, these particles were crushed into small fragments and deposited on holey carbon films for TEM observations.

\section{Rhombohedral - icosahedral relationships.}

As previously demonstrated, the dodecahedral particles obtained by slow solidification show a microcrystalline structure readily recognizable on high resolution imaging, where the product appears structured in domains of less than $20 \mathrm{~nm}$ in size (e.g. Fig. 1a and c and see Ref. [8]). Its pseudo-icosahedral symmetry, deduced from electron diffraction patterns, is angularly in accordance with the icosahedral symmetry group but not the characteristic reflection distances. The rhombohedral structure of such material has been identified by reconstructing its 3-dimensional lattice from different 2-dimensional lattice sections observed on high resolution images of 5-, 3and 2 -fold zone axes [8]. This rhombohedral cell of parameters $a=32.16 \AA, \alpha=36^{\circ}$ corresponds to an approximant structure of the icosahedral phase and can be generated by cut and projection method of a 6D F-hypercubic lattice [14]. Its close relationship with the quasicrystalline phase, observed after transformation at high temperature, can be pointed out from a related larger facecentered rhombohedral cell of parameters $a=37.81 \AA, \alpha=63.43^{\circ}$ (Fig. 1b), which is identical to the Ammann prolate rhombohedron. In that case, the $a$ parameter is also equal to $2 \tau^{3}(4.46) \AA$, where the value of $4.46 \AA$ corresponds to the smallest characteristic parameter of the icosahedral state and $\tau^{3}$ to a specific inflation of icosahedral quasilattice $(\tau$ is the golden mean $(1+\sqrt{5}) / 2)$; the factor 2 can be related to the F-superstructure order of the icosahedral phase [3, 14]. It results that this approximant structure can be decorated with Ammann prolate and oblate rhombohedra of edge length $4.46 \AA$ (Fig. 1 b): the face marking of both rhombohedra by 3 white and 3 shaded faces, related by a 3-fold symmetry, is typical of the F-superstructure order; only faces of the same type are joined together in the construction. This constitutes a framework of stellate and triacontahedral polyhedra of icosahedral symmetry for the crystallographic structure of the phase, 

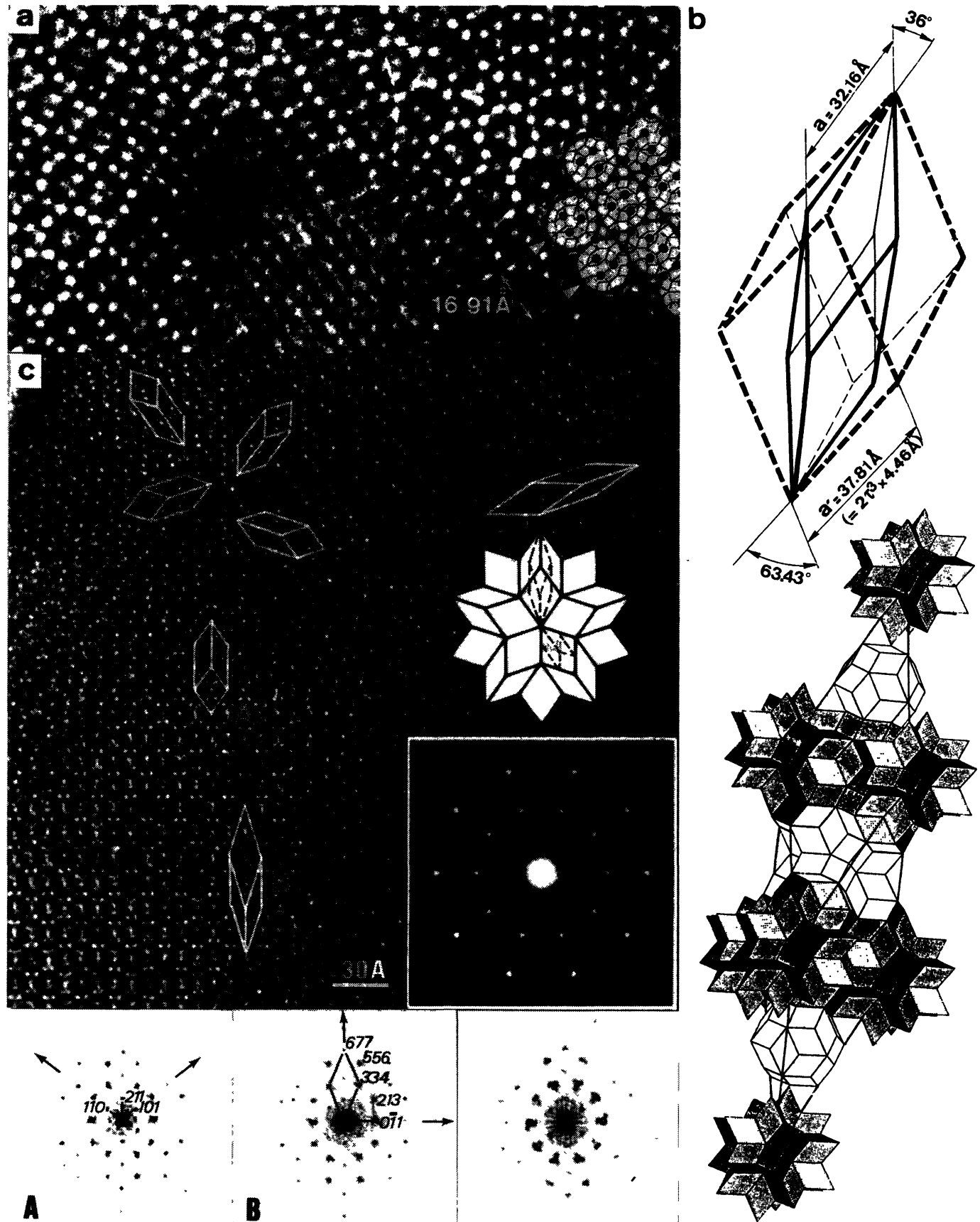

Fig. 1. - Characteristics of the microcrystalline structure of slowly solidified $\mathrm{Al}-\mathrm{Fe}-\mathrm{Cu}$ dodecahedral particles: a) HREM micrograph related to an orientation of 5-fold zone axis; b) relationships between both rhombohedral representations and arrangement of Ammann rhombohedra decorating the "approximant" rhombohedral structure; c) orientational relationships of crystalline domains (see text). 
from which, an arrangement of atomic clusters of perfect or nearly perfect icosahedral symmetry could be envisaged, similarly to the case of the $\mathrm{R}-\mathrm{Al}_{5} \mathrm{CuLi}_{3}$ cubic phase [15]. However, such a framework cannot be related to the HREM micrograph of the figure 1a, where rings of ten white dots surrounding three other white dots are observed. The arrangement of white dots seems to be rather related to $2 \mathrm{D}$-Penrose tiling where the tile edge length is $3.07 \AA\left(=\left(4.46 \tau \sin 63.43^{\circ}\right.\right.$ $\left.\left.\cos 18^{\circ}\right) / 2\right)$, instead of $3.99 \AA\left(=4.46 \sin 63.43^{\circ}\right)$ as it must be expected in the case of the projection of a decoration of Ammann rhombohedra of edge length $4.46 \AA$, a point of interest as advocated further in this paper.

Rhombohedral microcrystals exhibit special orientational relationships which display an overall pseudo-icosahedral symmetry and, thanks to a careful HREM study [8], it has been shown that the different orientations of the rhombohedral cells are those of the 20 rhombohedra in a stellate polyhedron. For instance, the relative orientations of the rhombohedral cell determined on the HR micrograph of pseudo icosahedral 5-fold zone axis (Fig. 1c) can be compared to those deduced from the stellate polyhedron. The zone axes of observation for rhombohedral domains are of two types, rational [111] and irrational $[(\overline{\tau+2} / \tau) 11]$; they are in good agreement with indexing of Fourier transform patterns obtained by using a computer image analyser. Note, along the irrational zone axis, the FT pattern is interpreted as a superimposition of several reciprocal sections very close to the irrational section and which zone axes are the rational approximant $[\overline{2} 11]$, [733] and [ $\overline{11} 55]$ of $[(\overline{\tau+2} / \tau) 11]$.

Previous studies have also shown that the microcrystalline to quasicrystalline transformation occuring between $650^{\circ} \mathrm{C}$ and about $850^{\circ} \mathrm{C}$, via different intermediate states preserves the same orientation of the icosahedral symmetry group. The figure 2 , borrowed to the reference [8a], corresponds to the in situ observation of this phase transition produces by electron beam irradiation heating, where it can be also noticed that apparently any morphological change of the specimen fragment has not occurred.

\section{Icosahedral quasilattice parameter.}

Observed by HREM, the icosahedral phase does not appear entirely perfect: a few jag fringes, typical of a disorder resulting of phason [6] can be observed on the micrograph of the figure 3, where the structure is oriented following a 5-fold zone axis. It may be pointed out on this micrograph that some features are similar to those observed on the rhombohedral structure. In effect, rings of ten white dots surrounding three other white dots are of the same size as those previously observed on the rhombohedral structure. A first interpretation of this HR micrograph can be proposed when considering a $2 \mathrm{D}$-Penrose tiling, where both rhomb tiles are decorated such that the normal matching rules for a hierarchy of expansion ratio $\tau$ is respected $[6,16]$. Thus, one generates a decorated $2 \mathrm{D}$-quasilattice which looks quite similar to the pattern of white dots observed on the HR micrograph. The rhomb tile edge length is equal to $3.07 \AA$ and corresponds to the smallest value which can be defined since each tile shares only one black point with neighbours.

However, the orientation of this 2D-quasilattice does not agree that of the icosahedral symmetry: there is a rotation of $18^{\circ}$ around the zone axis of observation, meanning that such a $2 \mathrm{D}$ quasilattice cannot be representative of a projection of 3D-quasilattice for the icosahedral phase. In order to solve this problem, it must be considered that the characteristic icosahedral quasilattice parameter is undetermined by an inflation factor $\tau^{n}$ ( $n$ integer), i.e. $4.46 \tau^{n}$, for a F-hypercubic lattice [14]. In that case, it is possible to consider the projection of an inflated icosahedral quasilattice commensurable to the $2 \mathrm{D}$-quasilattice which parameter and orientation can be in agreement with those of the icosahedral phase (Fig. 4a). A priori, the commensurable projection of a 3D- 

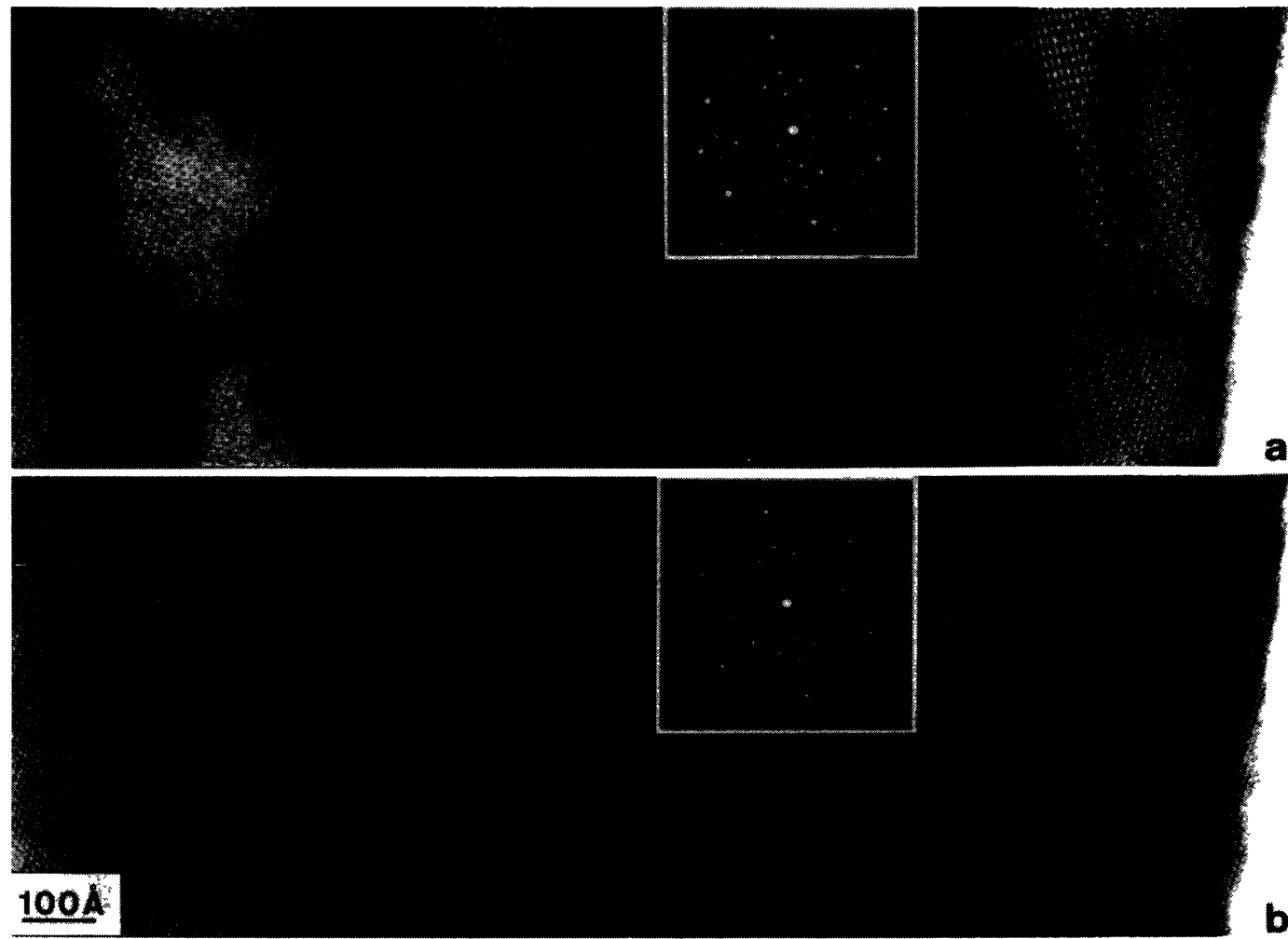

Fig. 2. - Crystalline to quasicrystalline transformation resulting, in the present case, of a direct in situ electron beam irradiation heating, allowing to observe that transition by HREM: a) microcrystalline structure oriented along a pseudo-icosahedral 2 -fold zone axis and b) perfect or nearly perfect icosahedral structure, characteristic of a face-centered-hypercubic system, obtained after transformation (from Ref. [8]).

quasilattice can be only defined for an inflation order $n \geq 3$. Nevertheless, we have found that it was rather ambiguous to precise the icosahedral parameter value for the $\mathrm{AlFeCu}$ phase, i.e. its inflation order, only from the decoration of white spot rings observed on the HREM micrograph of the figure 3 .

The inflation order of the quasilattice, which could be associated to this AlFeCu icosahedral phase, can be determined from HR images characteristic of the superstructure resulting of a face centered hypercubic lattice. Cahn, Shechtman and Gratias [14] have demonstrated that a reciprocal section of two-fold zone axis exhibits several reflections of surstructure with respect to reflections observed for a primitive hypercubic lattice, when the icosahedral structure is typical of a F-hypercubic lattice. Such a surstructure have been effectively identified by electron diffraction in the icosahedral $\mathrm{AlFeCu}$ phase [3-5]. In the direct space, the Ammann tiling related to the F-surstructure is bipartite, i.e. it can be splited into two types of vertices, decorated either by two different atoms or atomic clusters (see insert of the Fig. 5). Its projection following a two-fold zone axis is such that the two types of vertices do not superimpose and can therefore be distinguished. A HREM micrograph of two-fold zone axis of observation must therefore be also characteristic of this type of projection for a specific inflation order of the parameter $4.46 \AA$. We have checked this on the HR image of the figure 5, where the projection of a bipartite decoration of white and black dots of the Ammann tiling can be defined only from a quasilattice parameter equal to $4.46 \tau^{4} \AA$. 


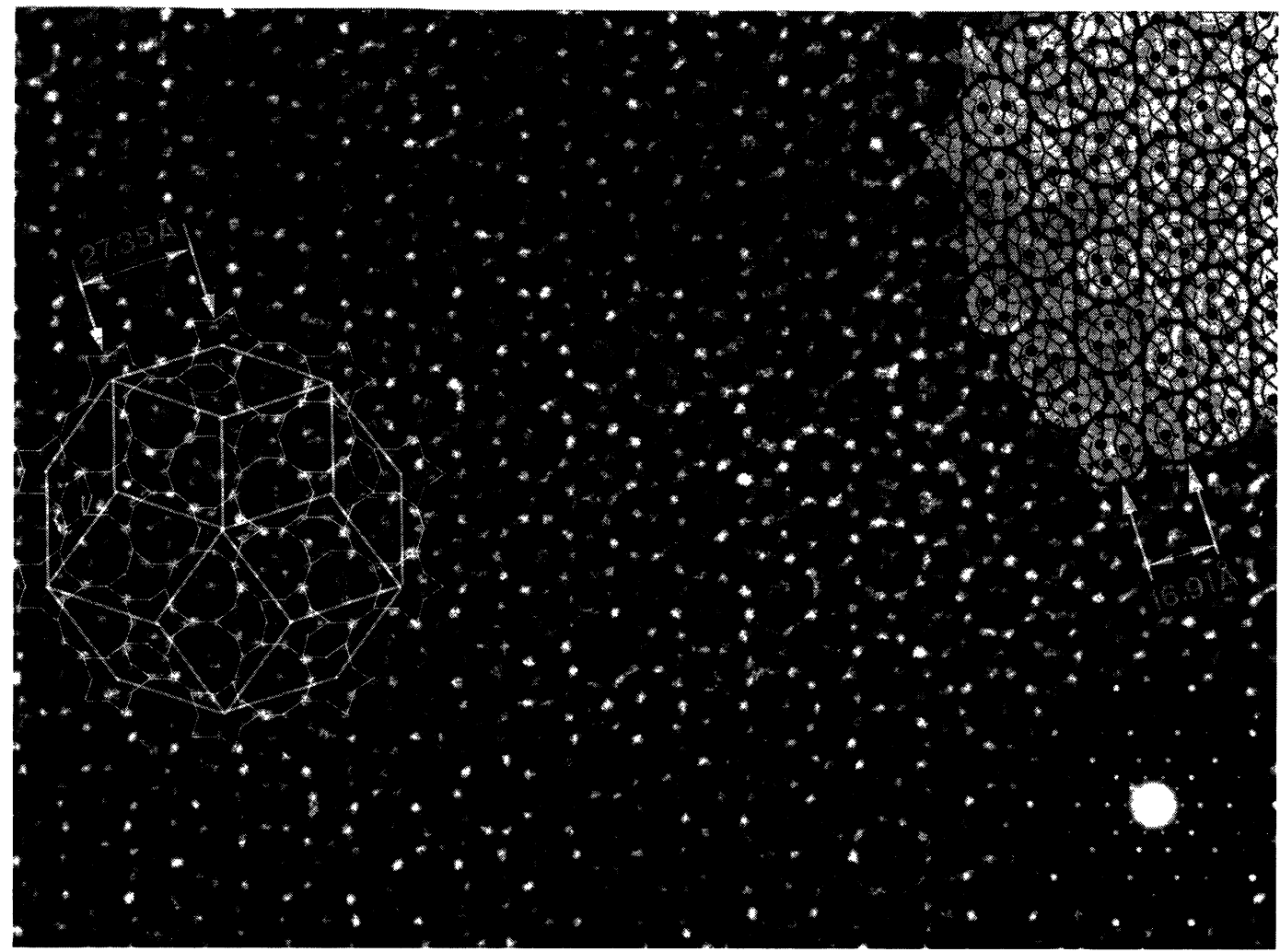

Fig. 3. - HREM micrograph of the Al-Fe-Cu material of icosahedral structure oriented following a 5-fold zone axis: star and rings of ten white dots surrounding three other white dots appearing on this HR image are quite similar to those deduced from a decorated 2D-Penrose tiling but with an orientation which do not agree that observed for the corresponding icosahedral symmetry group. However, further results, presented in the text and in figure 5 allow to consider the projection of an inflated quasilattice of parameter $4.46 \tau^{4} \AA$ $\left(=27.35 \AA / \sin 63.43^{\circ}\right.$, where $\sin 63.43^{\circ}$ corresponds to a projection factor of the Ammann rhombohedra edge length).

\section{Transition by phasons.}

Returning to the HREM micrograph of the figure 3, a projection of the framework decorating the " $4.46 \tau$ " quasilattice, viewed along a 5-fold zone axis, can then be suggested; it is outlined with white lines on the micrograph. Such a decoration of 2D-tiling does not imply, however, matching rules which enforce the quasiperiodicity (Fig. $4 \mathrm{~b}$ and c). Note that in 2 dimensions this decoration is related, through short displacements of black dots, or phasons, to the previous decoration of the 2D-Penrose tiling shown in figure 3. It may be also noticed that the representation of a decoration for the " $4.46 \tau^{4}$ " quasilattice has to be related to a single slice of Ammann rhombohedra; whether or not such a decoration remains invariante through thickness of several rhombohedra, is an unresolved question. In the same manner, differences between HR images of both icosahedral and rhombohedral states can be interpreted as resulting of the same type of displacement: the figure 6 


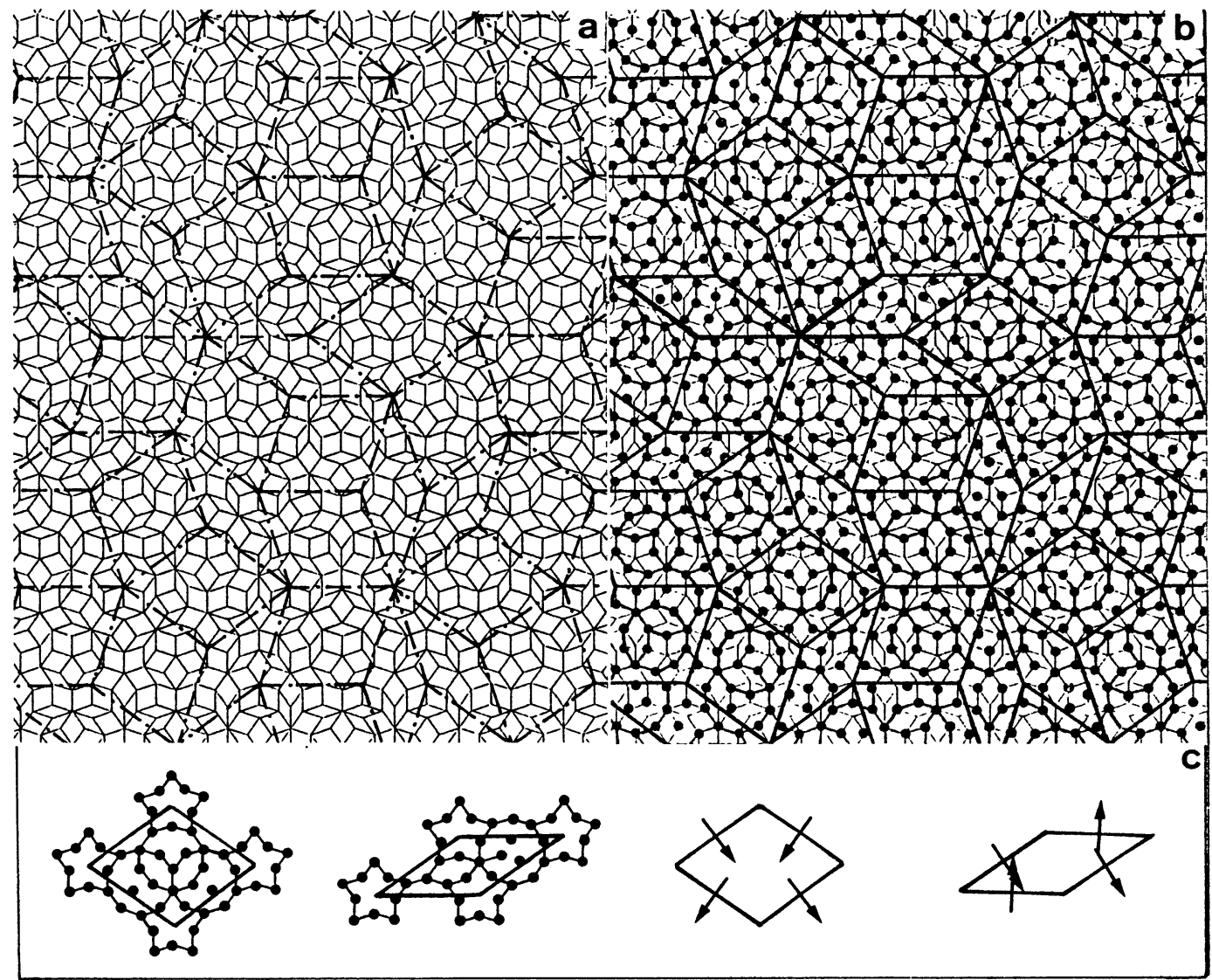

Fig. 4. - a) Projection of icosahedral quasilattice commensurated to a smaller 2D-Penrose tiling with a rotation of $18^{\circ}$ (see text); b) a decoration of the large tiling which can be related (i) through short displacements of black points (or dynamical phasons) to that of the one shown in insert of the figure 3 , and (ii) to the 3D quasilattice of parameter $4.46 \tau^{4} \AA$, found by HREM (Figs 5 and 3); c) elementary decoration of both tiles decorating the tiling shown in $b$ and related matching rules, which do not enforce the quasiperiodicity.

illustrates, for an idealization of the quasiperiodic image, that a limited crystalline domain (as it is observed by HREM) can be generated by phasons [6] from the quasicrystal; the relation between rhombohedral and icosahedral parameters being the same as the one experimentally observed, i.e. if the quasilattice parameter is equal to $4.46 \tau^{4}$, then the corresponding rhombohedral cell parameter is equal to $4.46 \tau^{3}$ (or $2 \times 4.46 \tau^{3}$ ).

Let's recall that various intermediate stages of this rhombohedral-icosahedral transition, as well as its reversibility, have been characterized by both direct in situ TEM heating experiments and thermal treatments of bulk dodecahedral particles [13]. One intermediate state has been identified as an icosahedral structure modulated along its 5-fold axes, with a pseudo period of modulation equal to $130 \AA$ which has been well interpreted from a dynamical phason condition applied to a model of atomic displacements defined within a 2D-1D cut projection scheme [13]. At present, a HREM study with computer image analysis of these intermediate states is in progress, in order to determine whether this modulated structure is characteristic of a phason or phonon field (or both). 


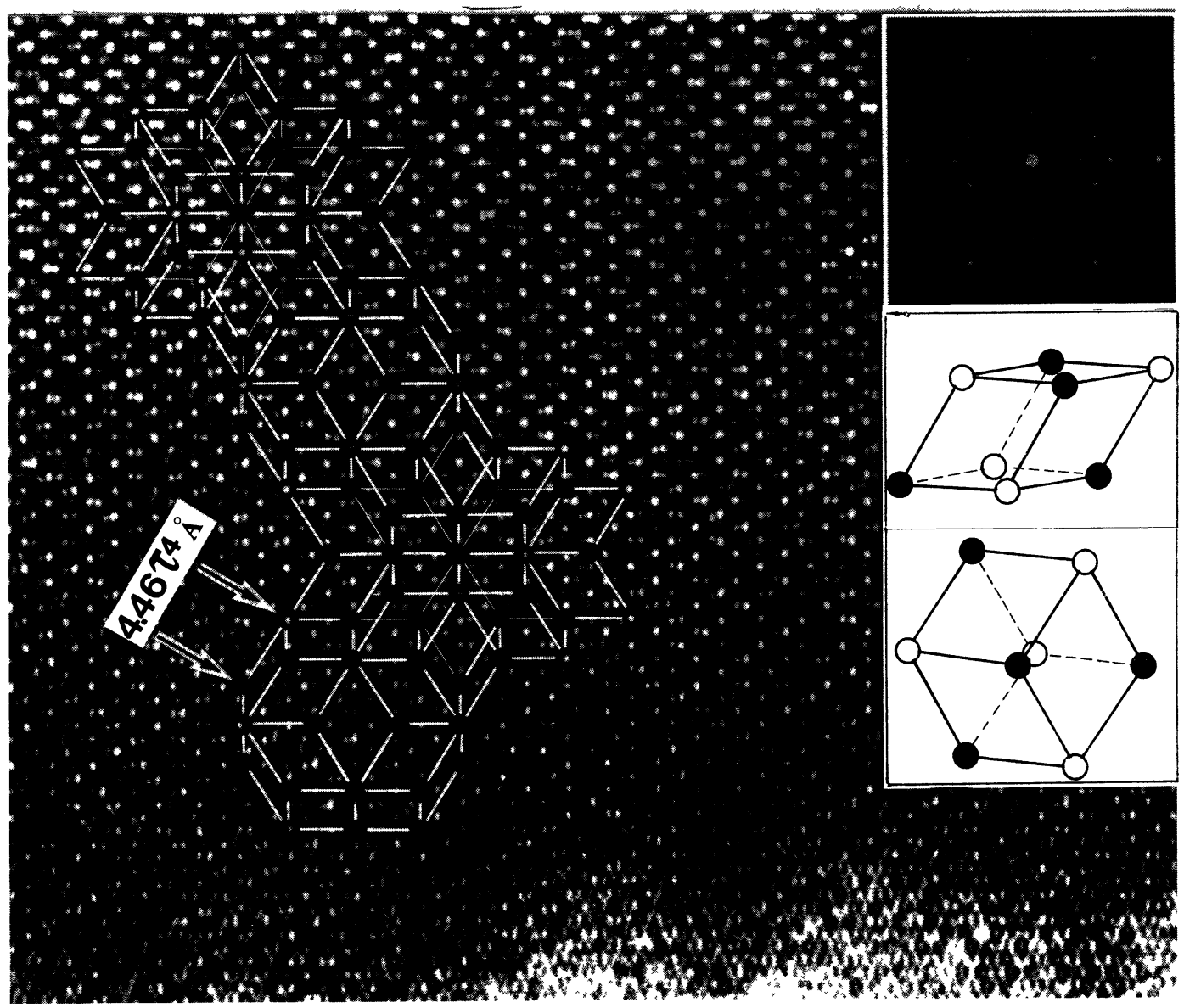

Fig. 5. - HREM micrograph of the icosahedral phase oriented following a 2-fold zone axis, where the quasilattice characteristic, typical of a F-hypercubic system, i.e. of a bipartite decoration, as illustrated in insert, can be checked for a quasilattice parameter equal to $4.46 \tau^{4} \AA$.

In the figure 7, is shown an example of HREM micrograph of the modulated icosahedral structure observed along a 5-fold zone axis. Due, a priori, to the Ewald's sphere curvature, satellite spots characteristic of the modulation along 5-fold axes, are observed on the corresponding electron diffraction pattern in the figure 7. Again, one can almost distinguish on the HR micrograph, rings of ten white dots surrounding three white dots, i.e. as for the icosahedral and rhombohedral states. Viewed at glance, such an image exhibits some jag fringes which can be characteristic of phason field, but also some distorted fringes, which would be rather characteristic of a phonon field.

\section{Concluding remark.}

We have precised, in this paper, some relationships between both the icosahedral and rhombohedral structures of a reversible phase transition found in the $\mathrm{Al}_{63.5} \mathrm{Fe}_{12.5} \mathrm{Cu}_{24}$ alloy. In particular, it has been pointed out that whether the icosahedral phase can be characteristic of a 3D-Ammann 
quasilattice, this last must be then considered with a quasilattice parameter equal to $4.46 \tau^{4} \AA$, i.e. with an inflation order $\tau^{4}$ instead of $\tau^{3}$ as it was, a priori, expected from the cell parameter $2 \times 4.46 \tau^{3} \AA$ of the related approximant rhombohedral phase.

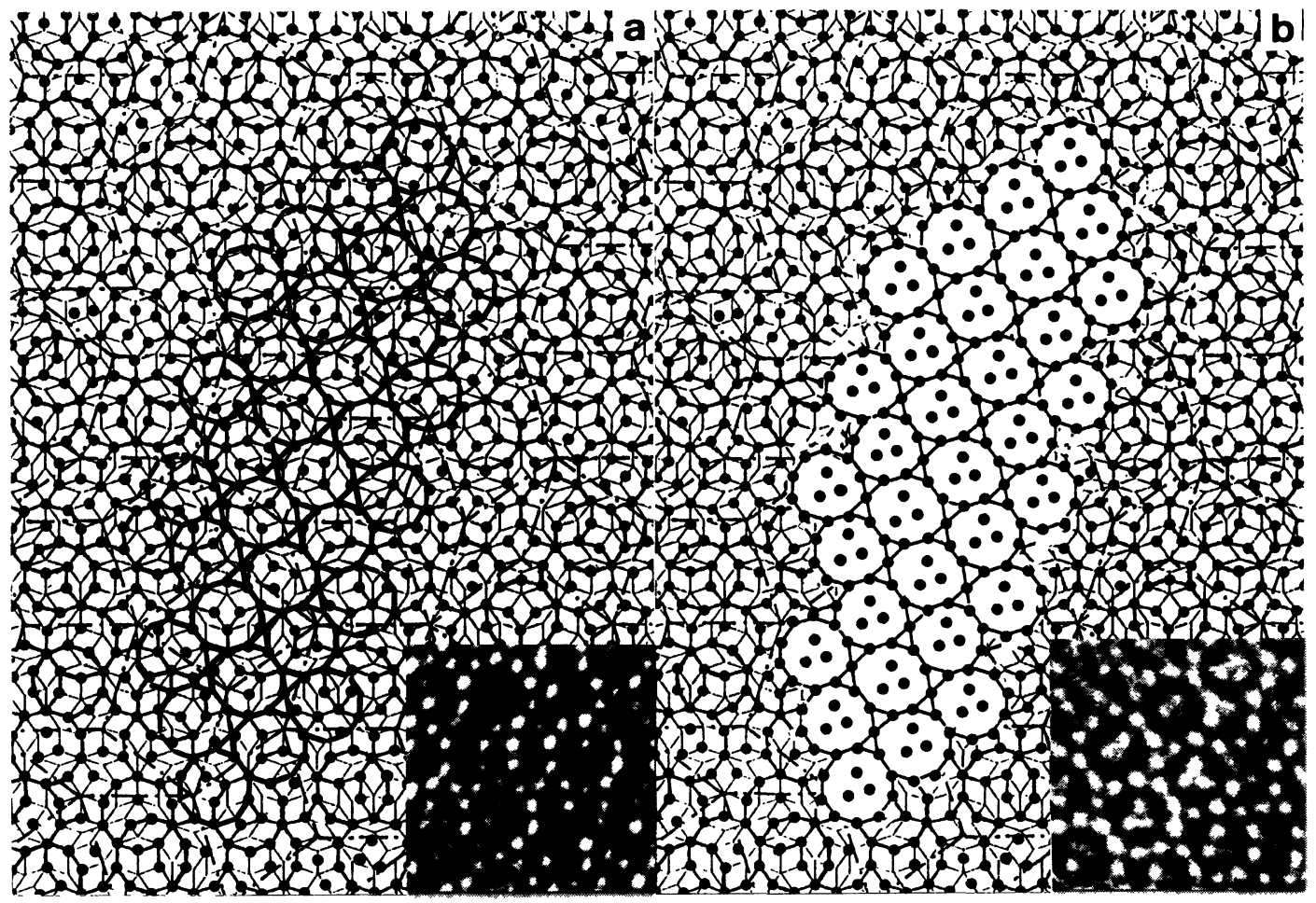

Fig. 6. - Model of crystallization of the icosahedral phase involving phasons, which allows to interpret the differences of features, observed by HREM, between icosahedral and rhombohedral phases.

For the phase transition, the consideration of 2D models deduced from HREM observations, has allowed to justify that structural changes result of phasons.

Finally from the discrepancy between the present results on the phase transition and those previously quoted in introduction [7], it may be suggested that whether the quasicrystalline phase can be characteristic of a deterministic quasilattice, their chemical composition and purity should be dependent on it. In such case, a slight departure from the critical composition of the very perfect icosahedral state could be a reason for the occurence of a phase transition. Further studies will be necessary in order to verify this assumption. 


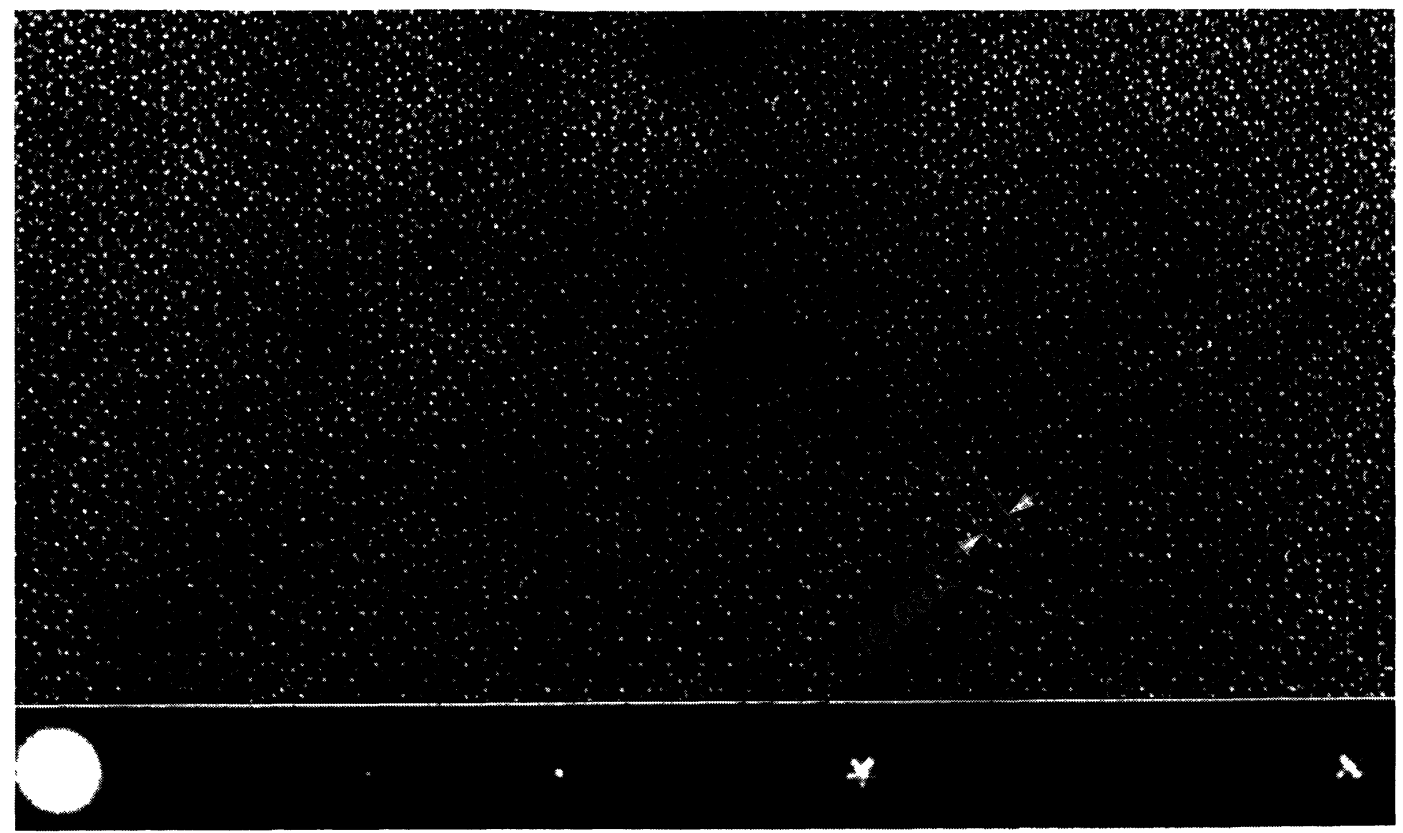

Fig. 7. - HREM micrograph of the modulated icosahedral structure observed along a 5-fold zone axis and detail of satellite spots exhibited by the corresponding electron diffraction pattern (the row of icosahedral reflections corresponds to a 2 -fold axis).

\section{References}

[1] Shechtman D., Blech I., Gratias D. and Cahn J.W., Phys. Rev. Lett. 53 (1984) 1951.

[2] TSAi A.P., Inoue A. and MatSumoto T., J. Mat. Sc. Lett. 6 (1987) 1403; Jpn J. Appl. Phys. 26 (1987) L1505 and J. Mater. Sci. Lett. 7 (1988) 322.

[3] Devaud-RzePSKI J., Quivy A., CalvayraC Y., CoRnier-Quiquandon M. and Gratias D., Philos. Mag. B 60 (1989) 855.

[4] EBAlARD S. and SPAEPEN F., J. Mater. Res. 4 (1989) 39 and Ibid. 5 (1990) 62.

[5] GuRian C.A., Goldman A.I., STEPHENS P.W., HiRaga K., TSAi A.P., InOUE A. and Matsumoto T., Phys. Rev. Lett. 62 (1989) 2409.

[6] The Physics of Quasicrystals, J.P. Steinhardt and S. Ostlund Eds. (World Scientific, 1987) and references therein.

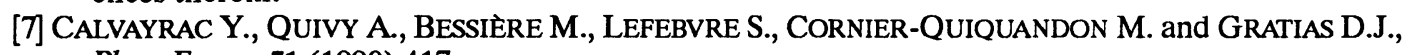
Phys. France 51 (1990) 417.

[8] AUDIER M. and GUYOT P., Proceedings of the III ${ }^{\text {rd }}$ Int. Conf. on Quasicrystals, Mexico (1989), J. Yacaman et al. Eds. (World Scientific, 1990) p. 288 and Proceeding of the $25^{\text {th }}$ Anniversary Adriatico Conf., ICTP, Trieste (1989), Jaric M.V. and Lundqvist S. Ed. (World Scientific, 1990) p. 74.

[9] BANCEL P.A., Phys. Rev. Lett. 63 (1989) 2741.

[10] JANot C., AUdiER M., de BoIssieu M. and Dubois J.M., Phys. Rev. Lett. (submitted).

[11] see for example, ELSER V., Phys. Rev. Lett. 54 (1985) 1730;

WIDOM M., STRANDBURG K. and SWENDSEN R.H., Phys. Rev. Lett. 58 (1987) 706;

WIDOM M., Proceedings of the 25 Anniversary Adriatico Conf., ICTP, Trieste (1989) M.V. Jaric and S. Lundqvist Eds. (World Scientific, 1990) p. 337.

[12] AUdier M., GUYOT P. and BRÉCHET Y., Philos. Mag. Lett. 61 (1990) 55.

[13] Audier M., BRECHET Y., de BOISSIEU M., GUYOT P., JANOT C. and Dubois J.M., Philos. Mag. (in press). 
[14] CAHN J.W., ShechtMan D. and Gratias D., J. Mater. Res. 1, (1986) 13.

[15] Audier M., PANnetier J., LeblanC M., Janot C., LANG J.M. and Dubost B., Physica B 153 (1988) 136.

[16] see for example OGAwA T., in Science on Form, Kato Y. et al. Ed., (KTK Sc. Pub. 1986) p. 479. 